\title{
Clinical implications of serum galactomannan measurement in patients with acute invasive fungal rhinosinusitis*
}

\author{
Hyun-Jin Cho ${ }^{1,2}$, Sang Duk Hong 3 , Hyo Yeol Kim³, Seung-Kyu Chung³, \\ Hun-Jong Dhong ${ }^{3}$ \\ ' Department of Otorhinolaryngology, Gyeongsang National University Hospital, Jinju, Republic of Korea \\ ${ }_{2}^{2}$ Institute of Health Sciences, Gyeongsang National University, Jinju, Republic of Korea \\ ${ }^{3}$ Department of Otorhinolaryngology - Head and Neck Surgery, Samsung Medical Center, Sungkyunkwan University \\ School of Medicine, Seoul, Republic of Korea
}

Rhinology 54: 336-341, 2016 DOI:10.4193/Rhino15.186

*Received for publication:

July 11, 2015

Accepted: March 31, 2016

\begin{abstract}
Background: Acute invasive fungal rhinosinusitis (AIFR) is an aggressive opportunistic infection with a high mortality rate. Recently, non-invasive techniques have been introduced for diagnosis of invasive fungal disease. The purpose of this study is to evaluate the diagnostic significance of serum galactomannan measurement in patients with AIFR.
\end{abstract}

Methodology: We conducted a retrospective case-control study of 28 patients with AIFR and 36 fungus ball (FB) patients. We evaluated clinical, laboratory, and pathologic findings along with disease course.

Results: In 28 patients with AIFR, there were 21 cases of invasive aspergillosis (IA) and 7 cases of invasive mucormycosis (IM). The control group was comprised of 36 patients with FB. The three-group analysis showed a statistically significant difference among the groups. At the cut-off value of 0.48 , the sensitivity and specificity were $71.4 \%$ and $93.0 \%$, respectively. Comparison of mean serum galactomannan levels in 5 non-survivors and 9 survivors at initial measurement showed no significant difference, but that became significantly different 1 week later. Statistical analysis showed that the levels of serum galactomannan decreased significantly according to the measurement-point in within survivor-group analysis. The difference in between survivor-groups analysis was also significant.

Conclusion: Serum galactomannan measurement seems useful for early diagnosis and discrimination of fungal species in patients with AIFR. In addition, clinical outcomes may be related to the levels and patterns of serum galactomannan, especially in IA. The appropriate measurement of galactomannan might be helpful in treating the patients at high risk for AIFR.

Key words: invasive fungal rhinosinusitis, galactomannan, aspergillosis, immunocompromised host, aspergillus antigen

\section{Introduction}

Acute invasive fungal rhinosinusitis (AIFR) is rare in immunocompetent individuals; however, the disease is often fulminant in immunocompromised patients, with very high mortality, ranging from $20 \%$ to $80 \%{ }^{(1,2)}$. Although there are many studies emphasizing the importance of early diagnosis, there are few reports demonstrating improvement in overall outcomes ${ }^{(3-6)}$. The diagnosis of AIFR is generally established with histopathologic features of invasive infiltration of fungus into the tissues and vascular structures ${ }^{(7,8)}$. However, it is often difficult to diagnose AIFR promptly in clinical practice. In 2002, a consensus group of the European Organization for Research and Treatment of Cancer (EORTC) and the National Institute of Allergy and Infectious Diseases Mycoses Study Group (MSG) proposed the definition of invasive fungal disease (IFD). Thereafter, EORTC/MSG revised the guideline in 2009. The growing interest in non-invasive techniques, such as high-resolution imaging or indirect diagnostic testing including detection of cell wall constituents or fungal 
DNA in serum or body fluid, have prompted revision of the definition of IFD ${ }^{(9,10)}$.

Galactomannan is a cell wall constituent of Aspergillus species and is spread to the bloodstream during fungal growth. Enzyme-linked immunosorbent assays (ELISA) were introduced to detect galactomannan more than 10 years ago. Thereafter, an abundance of clinical research has suggested a clinical role for the assay. Unfortunately, these studies were performed only in invasive pulmonary aspergillosis (IPA) and cerebral aspergillosis, and the results were controversial ${ }^{(11-13)}$.

To our knowledge, previous studies have been performed only in patients with major organ involved IFD. Furthermore, there has been no study elucidating the clinical role of galactomannan in patients with AIFR. The purpose of this study is to evaluate the applicability of serum galactomannan measurement in patients with AIFR. Our hypothesis is that the results may differ according to disease criteria and the diagnostic cut-off value.

\section{Materials and methods}

We undertook a case-control study at a single tertiary referral center. The case group consisted of consecutive patients with AIFR. Control group data were collected prospectively. After approval from the Institutional Ethics Committee (IRB no. 201401-030), medical records were reviewed to obtain clinical data for the analysis.

Since the galactomannan ELISA test had been introduced in our department in 2007, the study was conducted with patients who were treated in the department of otorhinolaryngologyhead and neck surgery between 2007 and 2014.

\section{Definition of patient group}

Twenty-eight consecutive patients with AIFR and 36 patients with fungus ball (FB) were allocated into the case and control groups, respectively. The diagnosis of AIFR was established according to EORTC/MSG criteria with clinically acute courses (less than 4 weeks).

Invasive aspergillosis (IA) and invasive mucormycosis (IM) were defined by pathologic diagnosis according to the results of hematoxylin and eosin stain, Periodic acid-Schiff stain, and Grocott's methenamine silver stain. All patients in the control group were pathologically confirmed to have FB caused by an Aspergillus species.

\section{Clinical and laboratory analysis}

Medical information including demographic data, underlying diseases compromising immune status, and radiologic and laboratory data was obtained. Microbiologic evidence was also investigated through fungus culture from peripheral blood and/ or sinus aspirates during surgery. Anatomical involvement of AIFR was also established from documented operative records and/or radiologic images, such as computed tomography and/ or magnetic resonance imaging. Clinical courses and antifungal regimens were also collected for analysis of prognosis. Survival was measured from initial diagnosis to death of the patient. Galactomannan antigen was measured using the sandwich microplate ELISA technique (Platelia ${ }^{\circledR}$ Aspergillus Ag, Bio-Rad Laboratories, Inc.). The test can be completed in several hours, and the results are reported as galactomannan index by comparison to a positive control. Galactomannan index is reported as a numerical outcome, and levels over a specific cut-off (range 0.5 to 1.5$)$ are regarded as positive results.

\section{Statistical analysis}

Comparisons of the difference in galactomannan levels among the groups were performed using the Kruskal-Wallis test. MannWhitney tests with Bonferroni correction were used for twogroup comparisons. Galactomannan index was analyzed using receiver operating characteristic $(\mathrm{ROC})$ curves and area under the curves (AUC) to obtain the optimal cut-off value. Sensitivity, specificity, and positive and negative predictive values were calculated with that cut-off. Analyses according to the clinical outcome were made using Mann-Whitney test. The relationship between initial and follow-up levels was analyzed using a generalized linear model. All statistical analysis was performed using SPSS 20.0 for Windows (IBM Corp., Armonk, NY, USA). A p-value less than 0.05 was considered statistically significant.

\section{Results}

Study polulation

A total of 64 patients were enrolled in the study. The characteristics of the patients are summarized in Table 1. The mean age was 61.0 years (range, $10-87$ years), and the male-to-female ratio was 27:37.

In 28 patients with AIFR, there were 21 cases (75\%) of IA and 7 cases (25\%) of IM. According to the EORTC/MSC criteria, 25 proven and 3 probable (due to fungal culture positive) diagnoses of AIFR were included. Twenty-seven of these 28 AIFR cases (96\%) developed in immunocompromised hosts, such as those with hematologic malignancy $(n=17)$, diabetes mellitus $(n=9)$, or other causes ( $n=9$ ) including systemic glucocorticoid use, chemotherapeutic drug, or organ transplantation. There was one immunocompetent patient who diagnosed with IM.

The control group was comprised of 36 patients with aspergilloma. There were 26 immunocompetent patients (72\%), and their mean age was 61.2 years (range, 35-87 years). Four patients (11\%) had diabetes mellitus, and 4 patients (11\%) had hematologic malignancy.

\section{Comparison of serum galactomannan}

Comparison among the three different groups was performed. The first group was AIFR caused by Aspergillus, or IA $(n=21)$. The remaining cases were assigned to the IM group $(n=7)$ and 
Table 1. Characteristics of the patients.

\begin{tabular}{|c|c|c|c|}
\hline Characteristic & $\begin{array}{l}\text { All Patients } \\
\qquad(\mathrm{N}=64)\end{array}$ & $\begin{array}{c}\text { AIFR } \\
(\mathrm{N}=\mathbf{2 8})\end{array}$ & $\begin{array}{l}\text { Fungus ball } \\
\qquad(\mathrm{N}=36)\end{array}$ \\
\hline Age, mean $\pm S D$ & $61.0 \pm 13.3$ & $60.6 \pm 15.7$ & $61.2 \pm 11.3$ \\
\hline (range), years & $(10 \sim 87)$ & $(10 \sim 80)$ & (35 87) \\
\hline \multicolumn{4}{|l|}{ Sex } \\
\hline Male & 27 (42\%) & $15(54 \%)$ & $12(33 \%)$ \\
\hline Female & 37 (58\%) & $13(46 \%)$ & $24(67 \%)$ \\
\hline \multicolumn{4}{|l|}{ Organism } \\
\hline Aspergillus & 57 (89\%) & $21(75 \%)$ & $36(100 \%)$ \\
\hline Mucor & $7(11 \%)$ & $7(25 \%)$ & $0(0 \%)$ \\
\hline \multicolumn{4}{|l|}{ Immune status } \\
\hline Competent & $27(42 \%)$ & $1(4 \%)$ & $26(72 \%)$ \\
\hline Compromized & 37 (58\%) & 27 (96\%) & $10(28 \%)$ \\
\hline \multicolumn{4}{|l|}{ Underlying disease } \\
\hline $\begin{array}{l}\text { Hematologic } \\
\text { malignancy }\end{array}$ & $21(33 \%)$ & $17(61 \%)$ & $4(11 \%)$ \\
\hline Diabetes mellitus & $13(20 \%)$ & $9(32 \%)$ & $4(11 \%)$ \\
\hline Other causes & $13(20 \%$ & $9(32 \%)$ & $4(11 \%)$ \\
\hline
\end{tabular}

AIFR $=$ acute invasive fungal rhinosinusitis.

the FB group $(n=36)$. Mean serum galactomannan levels were $0.83 \pm 0.46$ (IA group), $0.31 \pm 0.19$ (IM group), and 0.29 \pm 0.09 (FB group). The three-group analysis showed a statistically significant difference among the groups $(p<0.001)$. Additional comparisons between two of the three groups were performed. The difference between the IA and IM groups was significant $(p=0.017)$. A statistical significance was also found in the comparison between IA and FB $(p<0.001)$; however, there was no difference in the serum level of galactomannan between IM and FB $(p=0.96)$. The detailed results of comparison are summarized in Figure 1.

\section{Cut-off value, sensitivity, and specificity calculation}

The levels of serum galactomannan were significantly higher in the IA group than in the other groups. Further analysis using ROC (Figure 2) revealed 0.48 to be the cut-off value of maximal diagnostic power (AUC 0.870, $\mathrm{p}<0.001$ ). At the cut-off value of 0.48 , the sensitivity and specificity were $71.4 \%$ and $93.0 \%$, respectively. The positive predictive value and negative predictive value were $83.3 \%$ and $87.0 \%$, respectively (Table 2 ).

\section{Clinical symptoms and disease extents and serum galacto- mannan in IA group}

According to these results, serum galactomannan levels were elevated significantly in only IA group. In only IA group, comparisons according to the clinical symptoms, the results of endoscopic/radiologic exams and operative findings were performed.

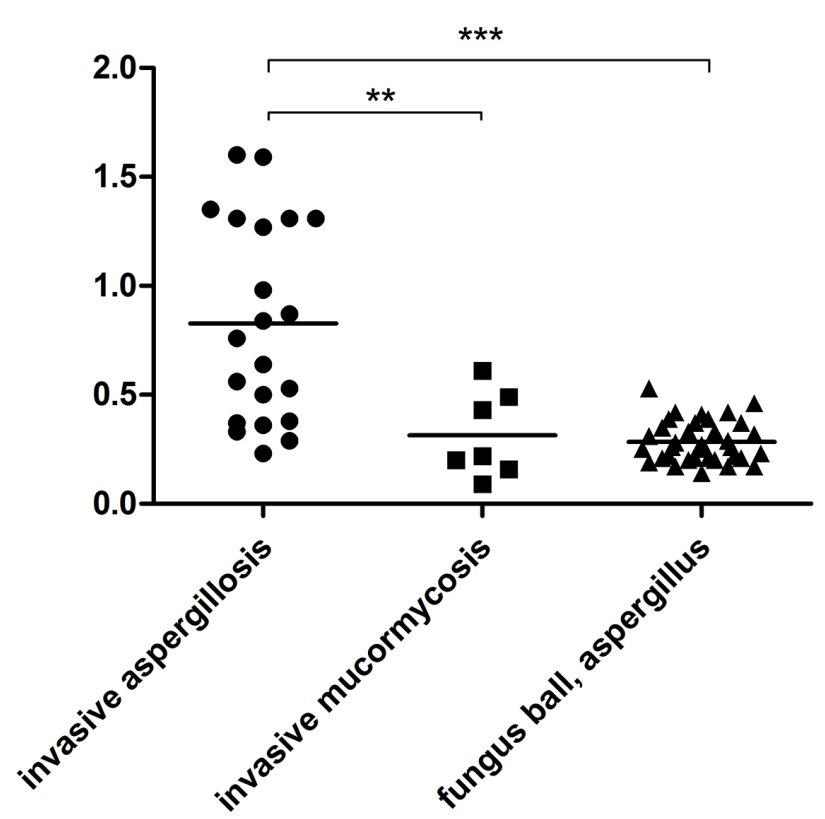

Figure 1. Comparison of serum galactomannan levels. Patients were assigned to invasive aspergillosis, invasive mucormycosis, and fungus ball, respectively. There are significant differences among the 3 groups. Additional analysis between each 2 groups shows statistically difference between invasive aspergillosis group and others. The serum galactomannan levels in each group were $0.83 \pm 0.46$ in invasive aspergillosis, $0.31 \pm 0.19$ in invasive mucormycosis, and $0.29 \pm 0.09$ in fungus ball. *** $p<0.001 ;{ }^{* *} p=0.017$.

The patients with cranial nerve palsy including optic nerve were defined central nerve system (CNS) involvement. The multiple subsites, extranasal involvements, and bilateral lesions were defined after obtaining the information of endoscopic, radiologic, and operative findings.

Among 21 IA patients, there were 6 (29\%) CNS involvements. Additionally, extranasal, multiple subsites, and bilateral involvements were identified in the frequency of $33 \%(n=7), 38 \%(n=8)$, and $9 \%(n=2)$, respectively. There was no significant difference

Table 2. Sensitivity and specificity of the serum galactomannan for diagnosis of invasive aspergillosis.

\begin{tabular}{|lccc|}
\multicolumn{5}{c}{} & \multicolumn{3}{c|}{ Invasive aspergillosis } \\
\multicolumn{1}{c}{$(+)$} & $(-)$ & Total \\
\hline Serum galactomannan & & & \\
\hline$>0.48$ & $15(83 \%)$ & $3(17 \%)$ & $18(28 \%)$ \\
$\leq 0.48$ & $6(13 \%)$ & $40(87 \%)$ & $46(72 \%)$ \\
\hline Total & $21(33 \%)$ & $43(67 \%)$ & $64(100 \%)$ \\
\hline
\end{tabular}

Sensitivity (\%): 71.43; Specificity (\%): 93.02; Positive predictive value (\%): 83.33; Negative predictive value (\%): 86.96 


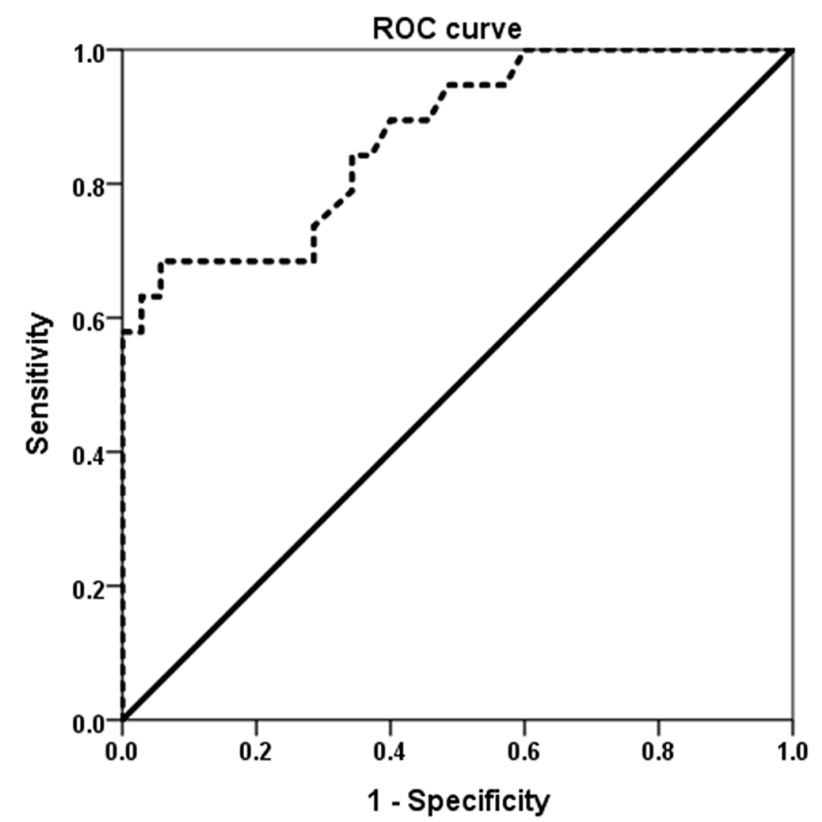

Figure 2. Receiver operating characteristic curve for the galactomannan assay. The cut-off value for diagnosis of invasive aspergillosis with the highest diagnostic power is 0.48 (Area under the curve $0.870, p<0.001$ ).

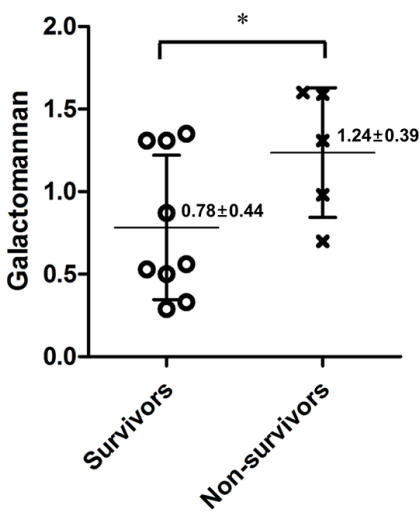

Initial

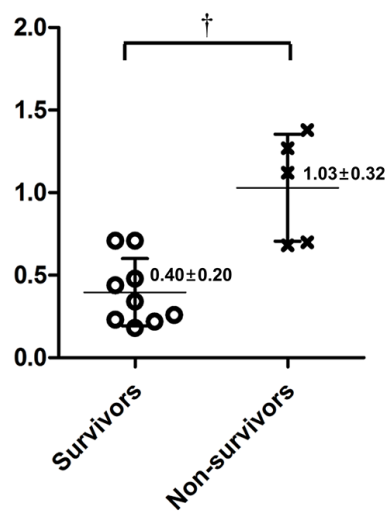

1 week
Figure 3. Clinical outcomes and serum galactomannan. Statistical analysis using a generalized linear model showed the levels of serum galactomannan decreased significantly according to the measurement-point in within survivor-group analysis $(p=0.022)$. In between survivor-group analysis, the difference of serum galactomannan levels was also significant $(p=0.005) .{ }^{*} p=0.083 ;+p=0.012 ;$ Mann-Whitney test.

$(p=0.614)$ in serum galactomannan levels in patients who had CNS involvement symptoms $(0.75 \pm 0.43)$ or not $(0.86 \pm 0.48)$. The comparisons of the serum galactomannan showed no significant differences, whether there were extranasal ( $1.03 \pm 0.32$ vs. $0.40 \pm 0.20, p=0.118)$, multiple subsites $(0.71 \pm 0.0 .42$ vs. $0.89 \pm 0.48$, $\mathrm{p}=0.385)$, and bilateral involvements $(0.96 \pm 0.45$ vs. $0.81 \pm 0.47$, $p=0.690$ ), or not.
Table 3. Summary of clinical outcomes in patients with invasive aspegillosis

\begin{tabular}{|c|c|c|c|c|}
\hline \multirow{2}{*}{$\begin{array}{l}\text { Case } \\
\text { (Age, sex) }\end{array}$} & \multicolumn{3}{|c|}{ Serum galactomannan } & \multirow[b]{2}{*}{ Survival } \\
\hline & Initial* & $f / u, 1 w k^{\dagger}$ & $\begin{array}{c}\text { Ratio } \\
(\mathbf{f} / \mathrm{u}: \text { initial })^{\ddagger}\end{array}$ & \\
\hline$F, 67$ & 0.5 & 0.48 & 0.96 & Alive \\
\hline$F, 77$ & 1.31 & 0.26 & 0.20 & Alive \\
\hline$F, 61$ & 0.29 & 0.18 & 0.62 & Alive \\
\hline$M, 52$ & 0.33 & 0.22 & 0.67 & Alive \\
\hline$M, 48$ & 1.31 & 0.71 & 0.54 & Alive \\
\hline$F, 56$ & 0.56 & 0.23 & 0.41 & Alive \\
\hline$M, 80$ & 0.87 & 0.44 & 0.51 & Alive \\
\hline$F, 59$ & 1.35 & 0.71 & 0.53 & Alive \\
\hline$M, 60$ & 0.53 & 0.34 & 0.64 & Alive \\
\hline Mean \pm SD & $0.78 \pm 0.44^{*}$ & $0.40 \pm 0.20 \dagger$ & $0.56 \pm 0.21 \neq$ & \\
\hline$F, 39$ & 1.6 & 1.38 & 0.86 & Dead \\
\hline$F, 61$ & 1.59 & 0.7 & 0.44 & Dead \\
\hline$F, 64$ & 0.98 & 0.68 & 0.69 & Dead \\
\hline$M, 51$ & 0.7 & 1.27 & 1.81 & Dead \\
\hline$F, 72$ & 1.31 & 1.12 & 0.85 & Dead \\
\hline Mean \pm SD & $1.24 \pm 0.39^{*}$ & $1.03 \pm 0.32 \dagger$ & $0.93 \pm 0.52 \ddagger$ & \\
\hline
\end{tabular}

${ }^{*} p=0.083 ;{ }^{\dagger} p=0.012 ;{ }^{\ddagger} p=0.112 ;$ Mann-Whitney test.

Clinical outcomes and serum galactomannan

Overall mortality rates of patients with AIFR, IA, and IM were $46.4 \%, 47.6 \%$, and $42.9 \%$, respectively. To analyze the relationship between the clinical outcomes and the value of galactomannan, we selected 14 patients with IA who had sufficient clinical and prognostic information. Additionally we divided the $14 \mathrm{IA}$ patients to survivors and non-survivors according to the survival outcome. Median follow-up period was 114 days (range, 5-661 days), and there were 5 deaths.

In 14 IA patients, mean serum galactomannan levels in non-survivors $(n=5)$ were higher tendency than those in survivors $(n=9)$, at both initial measurement $(1.24 \pm 0.39$ vs. $0.78 \pm 0.44, p=0.083$ ) and one-week follow-up ( $1.03 \pm 0.32$ vs. $0.40 \pm 0.20, p=0.012$ ), however there was statistically significant difference in only the follow-up measurement (Table 3, Figure 3).

However, statistical analysis using a generalized linear model showed that the levels of serum galactomannan decreased significantly according to the time points in within survivor-group analysis ( $p=0.022$ ). In between survivor-group analysis, the difference in serum galactomannan levels between survivors and non-survivors was also significant ( $p=0.005$ ) (Figure 3 ). Although there was no statistical significance, the ratio of initial to follow-up measurement showed a difference between groups $(0.93 \pm 0.52$ vs. $0.56 \pm 0.21)$. 


\section{Discussion}

The incidence of IFD appears to have increased, especially in the growing number of patients who are immunocompromised due to hematologic malignancies, diabetes mellitus, organ transplantation, systemic steroid therapy, and many other conditions causing immunologic alterations ${ }^{(1-3,14)}$. IFD can be generally subdivided according to the organ and kinds of pathogens involved. For example, IPA is the infectious lung disease caused by Aspergillus. Therefore, AIFR could be defined as acute infection caused by invasion of fungus into the tissue of the nose and paranasal sinuses. Despite previous studies investigating various prognostic factors, the clinical course of many patients with AIFR is likely disappointing ${ }^{(1,14-17)}$

Early diagnosis in patients with AIFR is essential for improved clinical outcomes. This could lead to prompt surgical resection of devitalized tissues and empirical administration of antifungal agents. Unfortunately, it is sometimes difficult to make the diagnosis of AIFR in clinical practice, even when pathologists are involved. Additionally, the accuracy of fungal culture is often disappointing. EORTC/MSC criteria have been developed and revised to overcome these problems ${ }^{(9,10)}$. By adopting the criteria, early detection and close observation are possible in high-risk patients. Unfortunately, there are few reports that applied the EORTC/MSC criteria for treating patients with AIFR, particularly in the field of otorhinolaryngology.

Aspergillus and Mucor are the two common fungal genera that cause AIFR. Both organisms account for more than $90 \%$ of AIFR and are ubiquitous in the environment. Discrimination between IA and IM is as important as early diagnosis of AIFR. Currently, newer antifungal agents, such as voriconazole and posaconazole, are available for solving the high incidence of amphotericin B-induced adverse effects ${ }^{(18,19)}$. In contrast to amphotericin B, which covers both Aspergillus and Mucor species, voriconazole and posaconazole have specific spectra of therapeutic activity. Moreover, there are some reports that show favorable positive effects of new antifungal drugs when combined with conventional agents ${ }^{(20-23)}$.

Galactomannan is a polysaccharide component present in the cell wall of Aspergillus species. The molecule is released hematogenously by fungal hyphae during tissue and vessel invasion. Galactomannan measurement using ELISA is commercially available and was approved by the United States Food and Drug Administration in 2003. Previous studies have found that measurement of serum galactomannan is useful for assessing the risk and monitoring the treatment response of immunocompromised patients. However, these studies included mostly IPA and cerebral aspergillosis with inconsistent results. Furthermore, the optimal cut-off level of galactomannan by ELISA has not yet been established ${ }^{(24-26)}$.

In the past, many studies have investigated the role of serum or body fluid measurement of galactomannan in patients with
IFD. Evidence from research for IPA and cerebral aspergillosis has some limitations. For instance, pathologic confirmation of IFD, the diagnostic gold standard, is often difficult. It is not always possible to procure a sufficient pathologic specimen from the primary site because of the high risk of obtaining the specimen from involved major organs as well as the elevated possibility of bleeding in these critically ill and thrombocytopenic patients. Our first objective was to discover the clinical role of serum galactomannan in the diagnosis and treatment of IA. To ascertain the biological rationale of our hypothesis that serum levels of galactomannan are affected by other types of fungal infections, we set invasive non-Aspergillus and non-invasive Aspergillus infections (IM and FB, respectively) as comparable control groups. As mentioned in the results section, serum levels of galactomannan were significantly higher in the IA group than in other groups $(p<0.001)$. In addition, the sensitivity and specificity of the test were $71.4 \%$ and $93.0 \%$, respectively. The positive and negative predictive values were both favorable (over $80 \%$ ). Moreover, we could obtain higher diagnostic values with adjustment of the cut-off value depending on the purpose of the test. In the clinical setting, we have experienced the confusing situation of attempting to discriminate between Aspergillus and Mucor. The diagnosis is often delayed or difficult in spite of adequately obtained specimens and clinical data. Given the results of this study, measuring the serum galactomannan is recommended for initial evaluation of patients at high risk for AIFR. In our result, serum galactomannan were elevated significantly in IA group, and we hypothesized that increased Aspergillus fungal burdens might be related to the higher levels. Therefore we compared the serum galactomannan levels according to CNS, extranasal, and extensive involvements, however, there was no significant relationship between those factors. Although there was no significance, extranasal involvement showed the tendency of higher serum levels ( $1.03 \pm 0.32$ vs. $0.40 \pm 0.20, p=0.118$ ). Future investigation could be elucidated about disease extents, fungal burden, and serum galactomannan levels. In addition, we compared the data according to the outcome of survival. There was a trend of mean galactomannan levels of non-survivors were higher than those of survivors at both initial and follow-up measurements, however there was no significant difference in initial measurement. Although there was inconsistent significance, there were differences in the levels and decreasing patterns of serum galactomannan in these two survivor-groups. Further statistical analysis found that there were significant differences in within survivor-group and between survivor-group comparisons ( $p=0.022$ and $p=0.005$, respectively).

The relatively small number of cases from a single center in this study might limit the generalizability of this clinical information. However, incidence of AIFR is quite low even in immunocompromised hosts, and this study evaluated 28 consecutive patients 
with AIFR, comparable to previous studies. As far as we know, this is the largest study of proven AIFR cases at a single institution and the first study to elucidate the clinical implications of serum galactomannan in association with AIFR. Additional studies are needed to further evaluate the clinical value of this topic in AIFR.

\section{Conclusion}

Galactomannan measurement in serum seems useful for early diagnosis and discrimination of fungal species in patients with AIFR. In addition, clinical outcomes may be related to the levels and patterns of serum galactomannan, especially in IA. The ap- propriate measurement of galactomannan might be helpful in treating the patients at high risk for AIFR.

\section{Authorship contribution}

Study concept and design: HJC, SDH, HJD; Acquisition of data, analysis an interpretation of data: $\mathrm{HJC}, \mathrm{SDH}$; Drafting of the manuscript: HJC; Critical revision of the manuscript: HJC, HJD; Study supervision: HJD, SKC, HYK

\section{Conflict of interest}

None to declare.

\section{References}

1. Monroe MM, McLean M, Sautter $\mathrm{N}$, et al. Invasive fungal rhinosinusitis: a 15-year experience with 29 patients. Laryngoscope. 2013; 123: 1583-1587

2. Duggal P, Wise SK. Chapter 8: Invasive fungal rhinosinusitis. Am J Rhinol Allergy. 2013, 27 Suppl 1: S28-30.

3. Rupa V, Maheswaran S, Ebenezer J Mathews SS. Current therapeutic protocols for chronic granulomatous fungal sinusitis. Rhinology. 2015; 53: 181-186.

4. Seo J, Kim HJ, Chung SK, et al. Cervicofacia tissue infarction in patients with acute invasive fungal sinusitis: prevalence and characteristic MR imaging findings. Neuroradiology. 2013; 55: 467-473.

5. DelGaudio JM, Clemson LA. An early detection protocol for invasive fungal sinusitis in neutropenic patients successfully reduces extent of disease at presentation and long term morbidity. Laryngoscope. 2009; 119: 180-183.

6. DelGaudio JM, Swain RE, Jr., Kingdom TT, Muller S, Hudgins PA. Computed tomographic findings in patients with invasive fungal sinusitis. Arch Otolaryngol Head Neck Surg. 2003; 129: 236-240.

7. Zhang F, An Y, Li Z, Zhao C. A novel model of invasive fungal rhinosinusitis in rats. Am J Rhinol Allergy. 2013; 27: 361-366.

8. Suslu AE, Ogretmenoglu O, Suslu N, Yuce OT, Onerci TM. Acute invasive fungal rhinosinusitis: our experience with 19 patients. Eur Arch Otorhinolaryngol. 2009; 266: 77-82.

9. De Pauw B, Walsh TJ, Donnelly JP, et al. Revised definitions of invasive fungal disease from the European Organization for Research and Treatment of Cancer/Invasive Fungal Infections Cooperative Group and the National Institute of Allergy and Infectious Diseases Mycoses Study Group (EORTC/MSG) Consensus Group. Clin Infect Dis. 2008; 46: 1813-1821.

10. Ascioglu S, Rex JH, de Pauw B, et al. Defining opportunistic invasive fungal infections in immunocompromised patients with cancer and hematopoietic stem cell transplants: an international con- sensus. Clin Infect Dis. 2002; 34: 7-14.

11. Pfeiffer CD, Fine JP, Safdar N. Diagnosis of invasive aspergillosis using a galactomannan assay: a meta-analysis. Clin Infect Dis. 2006; 42: 1417-1427.

12. Hope WW, Walsh TJ, Denning DW Laboratory diagnosis of invasive aspergillosis. Lancet Infect Dis. 2005; 5: 609-622.

13. Herbrecht R, Letscher-Bru V, Oprea C, et al. Aspergillus galactomannan detection in the diagnosis of invasive aspergillosis in cancer patients. J Clin Oncol. 2002; 20: 1898-1906.

14. Mistry SG, Kumar BN. The value of antifungal therapy in allergic fungal rhinosinusitis. Rhinology. 2014; 52: 9-18.

15. Turner JH, Soudry E, Nayak JV, Hwang PH. Survival outcomes in acute invasive fungal sinusitis: a systematic review and quantitative synthesis of published evidence. Laryngoscope. 2013; 123: 1112-1118.

16. Villarroel $M$, Aviles $C L$, Silva $P$, et al. Risk factors associated with invasive fungal disease in children with cancer and febrile neutropenia: a prospective multicenter evaluation. Pediatr Infect Dis J. 2010; 29: 816-821.

17. Kasapoglu F, Coskun H, Ozmen OA, Akalin $H$, Ener B. Acute invasive fungal rhinosinusitis: evaluation of 26 patients treated with endonasal or open surgical procedures. Otolaryngol Head Neck Surg. 2010; 143: 614-620.

18. Hamill RJ. Amphotericin B formulations: a comparative review of efficacy and toxicity. Drugs. 2013; 73: 919-934.

19. Saliba F, Dupont B. Renal impairment and amphotericin B formulations in patients with invasive fungal infections. Med Mycol. 2008; 46: 97-112.

20. Pagano L, Cornely OA, Busca A, et al. Combined antifungal approach for the treatment of invasive mucormycosis in patients with hematologic diseases: a report from the SEIFEM and FUNGISCOPE registries. Haematologica. 2013; 98: e127130.

21. Garbati MA, Alasmari FA, Al-Tannir MA, Tleyjeh IM. The role of combination antifungal therapy in the treatment of invasive aspergillosis: a systematic review. Int J Infect
Dis. 2012; 16: e76-81.

22. Lehrnbecher T, Attarbaschi A, Duerken M et al. Posaconazole salvage treatment in paediatric patients: a multicentre survey. Eur J Clin Microbiol Infect Dis. 2010; 29: 1043-1045.

23. Mukherjee PK, Sheehan DJ, Hitchcock CA, Ghannoum MA. Combination treatment of invasive fungal infections. Clin Microbiol Rev. 2005; 18: 163-194.

24. Vehreschild JJ. As galactomannan disappoints, our quest for a feasible diagnostic standard for invasive aspergillosis continues. Am J Respir Crit Care Med. 2014; 190: 248-249.

25. Maertens J, Buve K, Theunissen K, et al. Galactomannan serves as a surrogate endpoint for outcome of pulmonary invasive aspergillosis in neutropenic hematology patients. Cancer. 2009; 115: 355-362.

26. Rohrlich P, Sarfati J, Mariani P, et al. Prospective sandwich enzyme-linked immunosorbent assay for serum galactomannan: early predictive value and clinical use in invasive aspergillosis. Pediatr Infect Dis J. 1996; 15: 232-237.

Hun-Jong Dhong

Department of Otorhinolaryngology

- Head and Neck Surgery

Samsung Medical Center

Sungkyunkwan University School of Medicine

81 Irwon-ro, Gangnam-gu

Seoul 135-710

Korea

Tel: +82 234103579

Fax: +82 234103879

E-mail address: hjdhong@skku.edu 